BMJ Open

Respiratory

Research

\section{Investigating significant health trends in idiopathic pulmonary fibrosis (INSIGHTS-IPF): rationale, aims and design of a nationwide prospective registry}

To cite: Behr J, Hoeper MM, Kreuter $\mathrm{M}$, et al. Investigating significant health trends in idiopathic pulmonary fibrosis (INSIGHTS-IPF): rationale, aims and design of a nationwide prospective registry. BMJ Open Resp Res 2013;1:e000010.

doi:10.1136/bmjresp-2013000010

Received 4 October 2013 Accepted 25 November 2013

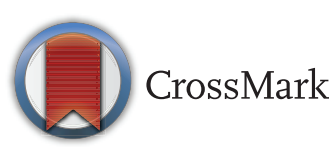

For numbered affiliations see end of article.

Correspondence to Prof. Dr Juergen Behr; j.behr@asklepios.com

\section{ABSTRACT}

Background: Guidelines on the diagnosis and management of idiopathic pulmonary fibrosis (IPF), a rare manifestation of chronic progressive fibrosing interstitial pneumonia, have been updated by ATS/ERS/ JRS/ALAT in 2011. In Europe, data are limited on the characteristics and management of such patients.

Methods/design: Investigating significant health trends (INSIGHTS)-IPF is a prospective observational longitudinal registry designed to describe the characteristics and management of newly diagnosed (incident) and prevalent patients with IPF on the long term. The registry uses a non-probability sampling approach to collect data on characteristics, therapeutic interventions, health-related quality of life and health economic parameters. At least 500 patients in ambulatory care will be included consecutively in about 30 centres. The study has been initiated in November 2012, and currently (December 2013) follows 344 patients. ClinTrials.gov identifier is NCT01695408. Discussion: INSIGHTS-IPF documents one of the largest IPF cohorts in Europe. The registry is expected to provide much-needed data on the characteristics and management situation of patients with IPF in Germany. It will allow comparisons with other countries. Gap analyses based on current guidelines for management of these patients will be possible.

\section{BACKGROUND}

\section{Definition}

Idiopathic pulmonary fibrosis (IPF) is defined as a specific form of chronic, progressive fibrosing interstitial pneumonia of unknown cause, occurring primarily in older adults, limited to the lungs. ${ }^{1}$ It is characterised by progressive worsening of dyspnoea and lung function and is associated with a poor prognosis. As stated in an international consensus statement jointly issued by ATS/ ERS, JRS and ALAT, IPF is a distinct clinical entity associated with the histological and/or

\section{KEY MESSAGES}

One year after initiation, the INSIGHTS-IPF registry has become one of the largest registries in the IPF indication in Europe.

- INSIGHTS-IPF will provide much-needed data on clinical history and the natural course of IPF under clinical practice conditions.

- The registry will provide insights into management related clinical outcomes and resource utilisation.

radiological appearance of usual interstitial pneumonia (UIP). ${ }^{1}$

The definition of IPF requires the exclusion of other forms of interstitial pneumonia including other idiopathic interstitial pneumonias and interstitial lung disease (ILD) associated with environmental exposure, medication or systemic disease.

IPF predominantly presents in older individuals (cases in persons aged less than 50 years are rare), with preponderance in men and previous or current smokers. Patients present with unexplained chronic exertional dyspnoea, and commonly with cough, bibasilar inspiratory crackles and finger clubbing.

\section{Incidence and prevalence of IPF}

Overall, epidemiological data on the incidence and prevalence of IPF are limited. ${ }^{1}$

In a population-based study from New Mexico, the incidence of IPF was estimated at 10.7 cases $/ 100000 /$ year for men and 7.4 cases $/ 100000 /$ year for women. ${ }^{2}$ In the UK, an overall incidence rate of only 4.6/100 000 person-years was reported, however, with an estimated increase by $11 \%$ annually between 1991 and 2003 (not attributed to aging of 
the population or increased ascertainment of milder cases). ${ }^{3}$ In the USA, based on a large database of healthcare claims in a health plan, the incidence of IPF was estimated between 6.8 and 16.3/100 000 persons. ${ }^{4}$

Prevalence estimates for IPF, as assessed in England, Japan, New Mexico, Norway and Greece, have varied widely from 2 to 29 cases $/ 100000$ in the general population. ${ }^{2}{ }^{5-8}$ The wide range in these numbers is likely explained by the previous lack of uniform definition used in identifying cases of IPF, as well as by differences in study designs and populations. ${ }^{1}$ In the USA, a recent analysis based on healthcare claims yielded a prevalence estimate between 14.0 and 42.7/100 000 persons depending on the case definition used. ${ }^{4}$ It is unknown whether the incidence and prevalence of IPF are influenced by geographic, ethnic, cultural or racial factors. ${ }^{1}$

\section{Diagnosis of IPF}

According to the consensus statement jointly issued by ATS/ERS/JRS/ALAT in 2011, the diagnosis of IPF requires the following ${ }^{1}$ :

- Exclusion of other known causes of ILD (eg, domestic and occupational environmental exposures, connective tissue disease and drug toxicity);

- The presence of a UIP pattern on high resolution CT (HRCT) in patients not subjected to surgical lung biopsy;

- Specific combinations of HRCT and surgical lung biopsy pattern in patients subjected to surgical lung biopsy.

\section{Natural course of disease}

Data on the natural course of IPF are from retrospective and a few prospective studies, including the placebo arms of clinical trials (limited to short observation periods). The previously reported median survival time of 2-3 years from the time of diagnosis could be an underestimate, at least when accounting for patients with a preserved lung function. Notably, the natural course is unpredictable for a given patient at the time of diagnosis; while the majority of patients experience steady worsening (slow progression), others remain stable or have rapid progression of disease. ${ }^{9}{ }^{10}$ Acute respiratory worsening either due to secondary complications (eg, pneumonia, pulmonary embolism, pneumothorax or cardiac failure) or due to unknown reasons (in this case the term acute exacerbation is used) are suggested to occur in about $5-10 \%$ annually. ${ }^{1}$ A recent retrospective review of 461 patients with IPF has found 1-year and 3-year incidences of acute exacerbations of $14.2 \%$ and $20.7 \%$, respectively. ${ }^{11}$

\section{Therapy}

The ATS/ESR/JRS/ALAT guideline finalised at the end of 2010 and issued in 2011 stated: "The committee did not find sufficient evidence to support the use of any specific pharmacological therapy for patients with IPF."1

A number of drugs are used for the treatment of IPF, including steroids, azathioprine and $N$-acetylcysteine
(NAC; and sometimes the latter 3 in combination). However, recently the NHLBI (part of the National Institutes of Health) has stopped the triple-drug therapy arm consisting of prednisone, azathioprine and NAC of the US PANTHER trial due to safety concerns. ${ }^{12}$

The only drug that has received regulatory approval for the treatment of mild or moderate IPF in Europe and Japan is pirfenidone, a pyridone compound with pleiotropic, anti-inflammatory, antifibrotic and antioxidant properties, with antagonism of transforming growth factor- $\beta 1$ effects. ${ }^{13}$

As for supportive pharmacological and nonpharmacological therapy, the evidence is limited, too. This includes anticoagulants, long-term use of oxygen, mechanical ventilation and lung transplantation. ${ }^{1}$

New therapies are under development, including the tyrosine kinase inhibitor nintedanib. ${ }^{14}$ It is, therefore, conceivable that in the present registry new drugs for the treatment of IPF will be documented once they are available.

\section{Patient-related endpoints}

The assessment of quality of life (QoL) parameters are important patient-related outcomes (PROs). ${ }^{15}$ QoL improvements have a role in the approval process of new drugs, and also in the subsequent benefit assessment as part of the reimbursement procedure. ${ }^{16-18}$ For example, the National Institute for Clinical Excellence (NICE) in the UK, the Institute for Quality and Efficiency in Health Care (IQWiG) and the Joint Federal Committee (G-BA) in Germany consider health-related QoL in addition to mortality and morbidity as a central element to justify additional benefit of a given drug. ${ }^{18} 19$ Self-assessment of health may differ substantially from the assessment by the treating physician or other individuals, often due to coping with the limitations associated with the disease.

Various questionnaires are used in the IPF registry, some generic, some respiratory disease specific, but all validated in a German language version. Of the generic questionnaires (not disease specific), results can be compared with other indications or diseases, or the general US-American $^{20}$ or German population. ${ }^{21}$

\section{Economic aspects}

IPF is of high interest for the healthcare system as well as for payers when considering costs. However, data on economic issues are limited, while needed for assessing cost-effectiveness of interventions. ${ }^{22} 23$

The current IPF registry will be suitable for various cost analyses. All pharmacological and health economic parameters will be derived from either one or both of the following ways and in most cases subsequently priced:

- Direct assessment of the resource use by the clinician.

- Indirect assessment by adaptation of already recorded clinical data. 


\section{Rationale for the present registry}

Long-term data on the natural course of IPF are missing. Furthermore, there is a lack of information on IPF in terms of epidemiology (incidence and prevalence) and patient characteristics. While in IPF substantial efforts are being made to investigate the efficacy and safety of new drugs in controlled clinical trials, there is a lack of data on the situation and treatment of patients in clinical practice. In a call for action on an IPF registry by Wilson $e t a l^{24}$ in 2008, it was noted that improved survival from this disease is dependent on better understanding of the epidemiology of the disease, its diagnostic spectrum and an analysis of outcomes from emerging therapies at a significant level. Furthermore, a registry can document the introduction of new therapies and complement the data from randomised controlled trials.

Therefore, registries, surveys and epidemiological studies have gained a great importance in various diseases, for example, in cardiology, as numerous examples show. ${ }^{25-27}$ They are particularly suitable for quality assurance, as individual centres can compare their results with other centres and with what is stated in guidelines.

In Germany, two multicentre registries on IPF have been initiated in 2009, both coordinated by University of Giessen Lung Center. First, the 'European IPF Network: Natural course, Pathomechanisms and Novel Treatment Options in Idiopathic Pulmonary Fibrosis' (eurIPFnet ${ }^{28}{ }^{29}$ ). This translational research programme aims, among other scientific goals, to establish a European-wide, Internet-based registry (eurIPFreg) and biobank (eurIPFbank) for IPF. ${ }^{28}$ Second, the GOLDnet, which takes a broader approach and focuses on basic research in diffuse parenchymal lung diseases and the development of test systems in diagnosis and activity grading ${ }^{30}$; this registry has been terminated in 2012.

The present clinical IPF registry will have some similarities to the present registries (internet-based, overlap in certain documented parameters), but also have major differences. No biobank specimen will be collected, and the IPF diagnosis will not be adjudicated to obtain true real-life data. However, in the present IPF registry, drug utilisation and treatment patterns under clinical practice conditions will be documented in detail. Furthermore, PROs will be investigated in detail (eg, health-related QoL, caregiver burden).

\section{Study objectives}

\section{Overall objective}

The overall objective of the present IPF registry is to gain further insight on the characteristics and management of patients with IPF, as treated under real-life, clinical practice conditions. The project aims to provide information on disease characteristics (disease registry and burden of disease), treatment patterns (pharmacoepidemiology), long-term effects (outcomes registry), QoL and economic aspects.

In addition, in the postlaunch phase of nintedanib, the registry will optionally be extended and amended with an additional Case Report Form (CRF) module to serve the purpose to collect real-life data on treatment with nintedanib in the context of a postmarketing surveillance obligation if required. For this purpose, it is expected that at least 25 additional sites will be included.

So, this registry will be used to:

- Provide a comprehensive clinical picture of IPF;

- Provide an estimate of the incidence and prevalence of IPF (with the caveat that such data will be representative for expert centres, unless sites with low patient numbers will be invited for participation);

- Describe best supportive care prescribed in these patients for whom, according to the international IPF guidelines, ${ }^{1}$ no treatment with proven efficacy is available to date;

- Establish feasible investigation strategies (eg, use of study endpoints in the practice setting);

- Track access to healthcare and cost of caring for patients with IPF over time;

- Examine the implementation of the existing guidelines; improve access to the current treatment approaches and assist in the development of best practice guidelines;

- Adherence to IPF therapies;

- Optionally, collect detailed information of treatment with nintedanib after approval and marketing of the agent: the IPF registry will be capable to document prospectively data on nintedanib under real-life conditions. For this purpose, new variables may be added at a later stage based on an amendment as required (depending on the approval of the ethics committee).

\section{Specific objectives}

Baseline (cross-sectional part)

- Description of characteristics of patients with IPF in terms of

- key (socio-) demographic data including gender aspects;

- IPF risk factors, comorbidities;

- Methods used for IPF diagnosis;

- IPF disease severity and manifestation (including lung function, cardiopulmonary exercise testing and/or exercise capacity if available, laboratory values);

- IPF treatment modalities (detailed information on prescribed drugs and dose; non-pharmacological treatment; listing and score for lung transplantation).

- Assessment of PROs such as QoL.

\section{Follow-up (prospectively up to at least 2 years after} inclusion)

- Prospective observation of patients with IPF

- Clinical course of IPF (eg, in terms of symptoms, lung function and exercise capacity if available);

- Prospective collection of outcome data (such as acute respiratory worsening, exacerbations, hospitalisation due to any cause and due to IPF, other complications, lung transplantation and survival). 
- Documentation of treatment pathways (switch/ add-on/discontinuation of medication), and of nonpharmacological treatment (eg, start of long-term oxygen therapy and new listing for lung transplantation).

- Assessment of PROs such as QoL annually (for comparison with baseline).

- Assessment of treatment intensity, frequencies and resource use for pharmacoeconomic analyses.

\section{METHODS}

\section{Design and setting}

This IPF registry allows for structured, noninterventional collection of data. Participating physicians will not be subject to any instructions with regard to the diagnosis and therapy of their patients. All examinations performed depend on the discretion and clinical routine of the physician.

The registry contains a basic set of variables (mandatory data) which are essential for all included patients. Another set of variables (facultative data) can be requested from the participating sites but is left to their discretion. The platform is capable for substudies (ie, addition of further variables) in selected centres to facilitate research collaboration between institutions. The patients are informed about the study and data protection issues with written patient information, and they must provide written informed consent before they can be documented.

\section{Patients}

It is planned to include at least 500 patients fulfilling all of the following criteria:

- At least 18 years of age;

- IPF based on physician diagnosis.

No explicit non-eligibility criteria are defined to avoid selection of patients and thus violation of the 'real-life' principle. Patients will be included in a consecutive manner at each site in order to avoid selection bias.

\section{Eligible sites}

Physicians managing patients with IPF are eligible for participation. Approximately 15-20 sites across Germany are initially planned (with a possible increase to about 30 sites, to achieve country representativeness). In order to ensure adequate patient numbers per centre, and high quality of data, expert lung centres or practices will be involved.

A roll-out of the study to other centres and countries is possible. A minimum of five patients/year should be included per centre, to ensure that centres have adequate patient numbers to get accustomed to the study procedures. The number of patients per site is not limited. Thus, the study will likely be representative for expert centres in Germany.

As described, a further optional and specific extension of the registry is feasible after launch of nintedanib, if deemed necessary. In this case, at least 25 additional sites will be added.

\section{Schedule and variables}

The study schedule is displayed in table 1 . At baseline, a relevant patient history will be recorded including IPF-related events up to 12 months prior to this visit. Furthermore, the current status of the patient will be recorded in detail (see CRF in online supplementary appendix). As an optional assessment, QoL questionnaires will be completed by the patient, preferably in the physician's office.

At the follow-up visit every 6 months ( \pm 3 months), events within the period between baseline (or the previous follow-up visit) and the present visit will be recorded. As an optional assessment, QoL questionnaires will be completed by the patient, preferably in the physician's office. If the patient is not available for an on-site visit, the follow-up contacts can be performed via telephone, and the QoL sheets will be exchanged with the patient by mail.

\section{Extraordinary visits}

The following events if related to IPF must be documented in the database:

- Death;

- Hospitalisations;

- Clinical events (such as acute respiratory worsening including pneumonia, pulmonary embolism, pneumothorax, cardiac failure or acute exacerbations and lung transplantation);

- Change in medication for IPF or increase in treatment intensity (ie, add-on of drugs);

- Serious adverse events related to IPF medication.

Variables to be documented (if available), by observation time point, are listed in the table 1.

\section{Information on drugs}

This registry documents the management and treatment of patients with IPF in real-life clinical practice. The main aim of this registry is to document drugs used for IPF, in particular those drugs which have a recommendation according to the recent ATS/ERS/JRS/ALAT recommendation ('weak no' recommendation). ${ }^{1}$

Information regarding these drugs can be derived from the respective summary of product characteristics. The following drugs will be documented in detail in terms of drug name and dosage, including combinations of the mentioned drugs:

- Steroids

- Immunomodulators (azathioprine, cyclophosphamide, mycophenolate mofetil, etc)

- NAC

- Pirfenidone

- Anticoagulants 
Variable

Eligibility criteria

Baseline

months*

Physician-diagnosed IPF based on physician diagnosis

$\mathrm{X}$

Baseline information

(Socio-)demographic variables: age, gender, race, body mass index; employment status,

insurance status

(Potential) IPF risk factors

$x$

Cigarette smoking including pack years; environmental exposure; drug exposure;

gastro-oesophageal reflux; genetic factors (family history); other

\section{Comorbidities}

Atherothrombotic disease including coronary heart disease including previous myocardial

infarction),cerebrovascular disease, peripheral arterial disease; pulmonary hypertension;

emphysema, lung cancer, renal insufficiency, other diseases

IPF

Baseline information on IPF

First symptoms; date of first diagnosis; if performed, dates and results of HRCT, surgical lung

biopsy, bronchoalveolar lavage

Symptoms

Dyspnoea, cough fatigue, dizziness, chest pain, anxiety, etc

UCSD SOBQ ${ }^{33}$

Functional assessment

Lung function test (VCin, FVC, FEV 1 , TLC; $\left.\mathrm{DL}_{\mathrm{CO}} ; \mathrm{pO}_{2}, \mathrm{pCO}_{2}\right)^{42}$

6 min walk distance (if performed)

CPET (if performed)

Serological evaluation

Rheumatoid factor, anticyclic citrullinated peptide and antinuclear antibody titre and pattern $\dagger$

Others, for example, SCL-70, SS-A, SS-B

BNP/NT-pro BNP

IPF pharmacological treatment

Past/discontinued: drugs by class (eg, steroids yes/no)

Current: drugs by class;

Participation in IPF trial

Anticoagulation

Non-pharmacological treatment

Long-term-oxygen-therapy (liquid and/or concentrate)

Physician assessment

Physician's clinical rating of the probable course of IPF (stable, slow or rapid progression)

Quality of life (facultative: 1-2 times/year)

EQ-5D

Specific instruments for pulmonary disease

SGRQ

UCSD SoB

Management of IPF and physician contacts

Number of physician contacts (own office, other physicians)

Number and type of IPF-related procedures in last 6 months

Clinical events and hospitalisations

specifically due to: (acute) worsening of IPF, IPF-exacerbation; manifest pulmonary or

cardiovascular complications; lung transplantation; pulmonary rehabilitation

Number of days in hospital or in rehabilitation; work days lost due to IPF

\section{Survival status $¥$}

*Or extraordinary visit in case of events.

†As recommended in the ATS/ERS guidelines for distinguishing connective tissue disease from IPF.

$\ddagger$ Patients have been asked to provide consent to that they may be located or contacted once they have moved or changed centres.

$\mathrm{BNP}$, brain natriuretic peptide; CPET, cardiopulmonary exercise testing; $\mathrm{DLCO}_{2}$, diffusing capacity of the lung for carbon monoxide; FEV,

forced expiratory volume; FVC, forced vital capacity; IPF, idiopathic pulmonary fibrosis; $\mathrm{pCO}_{2}$, partial carbon dioxide; SGRQ, St George

Respiratory Questionnaire; UCSD SoB, University of California San Diego Shortness of Breath. 


\section{QoL questionnaires}

While physicians in daily routine assess health-related QoL in a non-standardised manner (usually by questioning the patient), in the context of this registry, they will be provided with validated questionnaires. Generally, questions in QoL questionnaires are not any more upsetting than those posed by a patient's physician or by friends and family members on a day-to-day basis. The use of the questionnaires is considered to be noninterventional. The patients are free to accept or refuse to fill in the proposed questionnaires in this study.

QoL questionnaires are available for download from the IPF registry website and are also provided to the centres in paper format. The centres are requested to send the filled questionnaires to the study centre in Dresden without delay, where the data will be entered in the database. The centres retain a copy of the QoL questionnaires which will be kept as part of the patient file; it can be used during the monitoring process (source data verification).

\section{Generic instruments}

\section{$E Q-5 D$}

The EuroQol (EQ-5D), developed by the EuroQoL group, is used most widely for general healthcare evaluation, including cost-utility evaluation. ${ }^{31}$ It consists of five domains (mobility, self-care, usual activities, pain or discomfort, and anxiety or depression) and a visual analogue scale (VAS) ${ }^{32}$ The scores range from 0 to 100 based on the level of health for each domain given by the participants. A score of 100 indicates that the current health is equivalent to full health; a score of 0 indicates that the current health is equivalent to death. According to the scores of the five domains, a sum utility score is calculated ranging from 0 to 1 . A score of 1 represents a perfect state. In addition, the patients have to rate their current health on a $20 \mathrm{~cm}$ vertical VAS scored from 0 to 100 reflecting the continuum from a best imaginable to the worst imaginable health state. The EQ-5D has proved to be a reliable, valid, selfreported measure.

\section{Specific questionnaires for pulmonary disease UCSD SOBQ}

The University of California, San Diego Shortness of Breath Questionnaire is a 24-item measure that assesses self-reported shortness of breath while performing a variety of activities of daily living. A new version has been shown to be a reliable and valid assessment tool in clinical practice and research in patients with moderateto-severe lung disease. ${ }^{33}$ Minimally clinically important differences have been reported. ${ }^{34}{ }^{35}$ This instrument has also been used in pirfenidone studies, allowing comparison and external validation of the results.

\section{St Georges Respiratory Questionnaire}

The St Georges Respiratory Questionnaire (SGRQ) is a self-completed questionnaire for measuring impaired health and perceived QoL in patients with chronic lung disease. A total of 76 items divided into three sections (symptoms, activity and impacts), from which an empirically derived weighted single score is calculated. As the score is independent of patient and study, size and change of score could have clinical meaning throughout the population. In IPF, SGRQ possesses a reasonable validity for differentiating individuals whose disease severity changes over time. ${ }^{36}$ The questionnaire has been validated in several languages including German. This instrument has also been used in pirfenidone studies, allowing comparisons with other studies and external validation of the results.

\section{Statistical methods}

Sample size was determined under feasibility aspects, and no formal sample size calculation was made.

A Statistical Analysis Plan (SAP) was set up prior to initiation of the registry that specified groups for analysis and the statistical methods. The observational study will be analysed descriptively, using established statistical and epidemiological methods. If inferential statistics such as CIs and/or $p$ values are calculated, the results will be regarded as descriptive only. No adjustments are made for multiple comparisons.

Continuous variables will be reported as median with interquartiles and other percentiles, and as mean with $\mathrm{SD}$, together with minimum and maximum values. Categorical variables will be reported as absolute and relative percentages.

For $\mathrm{AE}$ and $\mathrm{SAE}$ incidence, rates based on the number of included patients and incidence density based on the number of events/patient-years will be calculated. These events as well as (Serious) drug reactions will be presented overall and by MedDRA System Organ Class and Preferred Term. ${ }^{37}$

Data will be analysed with STATA version 13 (Stata Corporation, College Station, Texas, USA).

\section{Data collection}

Physicians enter the data of their patients directly in an Internet-based electronic hypertext-preprocessor case record form. At data entry, plausibility checks (eg, range checks, conditional checks, etc) will be performed. Data are transmitted using a Secure Sockets Layer via Internet. They are validated within the Content Management Systems Drupal, processed and eventually stored in a Structured Query Language (MySQL) database (developed by Oracle Corporation und Sun Microsystems). The documented patients and follow-up visits are only visible for the centre that entered the data. On a quarterly basis, data are centrally checked and, if needed, queries are sent to the centres. Monitoring visits with source data verification are performed in randomly selected centres.

QoL questionnaires will be completed on paper forms and will be entered in the clinical database by data managers in Dresden. 
Data management details will be described in a separate Data Management Plan.

\section{Statistical Analysis Plan}

All statistical methodology that will be applied will be described in detail in the SAP which will be finalised at the latest prior to database lock for the final analysis. The SAP will describe the analysis of the data collected in the eCRF (at baseline and at the 1-year follow-up visit) as well as the data obtained from the QoL assessments (EQ-5D, etc).

\section{Adverse events}

In this disease and outcomes registry, no special adverse drug reporting requirements apply. The physician has to report any adverse drug reactions in his/her routine ways as regular spontaneous reports in accordance with Volume 9A of The Rules Governing Medicinal Products in the EC as well as in accordance with local laws and regulations. Reporting sheets are available in the study file and for download on the registry website.

However, if in patients treated with nintedanib adverse events (non-serious or serious) occur, that is, any untoward medical occurrence irrespective of relation to the drug, physicians are obliged to report those to the Pharmacovigilance Department at Boehringer Ingelheim Pharma GmbH \& Co KG. Information is needed for reconciliation of such events with reports from clinical studies (event description, patient number in study and name of study).

\section{Methodological considerations and limitations}

The investigating significant health trends (INSIGHTS)IPF registry is prospective and recruits consecutive patients, thus limiting selection bias. It applies various measures for quality assurance, the most important one being on-site monitoring with systematic comparisons between study data and patient files. ${ }^{38} 39$

The main limitations of this study are those inherent to any registry. Given that this is an observational, nonrandomised study, different biases can obscure any true causal association. ${ }^{40}$ Clinical decisions of the treating physicians that may assign patients to different drugs based on disease severity, disease duration, presence of comorbidities and other factors can potentially introduce allocation or channelling bias and confound the association between treatment and outcomes. Survival in IPF cohorts is not only strongly influenced by clinical baseline characteristics and associated conditions, but also by the time interval between diagnosis and recruitment into the registry (survivor bias). Patients documented in the registry as prevalent cases could more likely have relatively stable disease and/or better response to IPF management compared with patients with newly detected disease (who in the case of rapid deterioration might not be included).

In our registry, there is no re-evaluation of patients, which may yield in some inclusion of patients with overlapping diagnoses, such as fibrosing non-specific interstitial pneumonia. However, as mainly IPF expert centres are involved, the quality of diagnoses should be high. Epidemiological nationwide data on the true incidence and prevalence of IPF will not be derived, but the registry will provide representative data on the situation of IPF expert centres and large pulmonary hospitals (referral centres) in which many of the known patients with IPF are treated.

\section{Intellectual property/governance}

The intellectual property of the study belongs to the INSIGHTS-IPF steering group (authors of this article). The legal sponsor of the study, GWT-TUD, Dresden, is responsible for the governance of the project.

\section{Documentation and archiving}

GWT-TUD is responsible for the archiving of the documentation for at least 10 years. Archived data may be held on electronic record, provided that a back-up exists and that hard copies can be obtained, if required.

The participating site has to care for the archiving of the identification list for at least 10 years or according to local legislation. There is no further obligation for archiving by the participating sites.

\section{Dissemination of information and publications}

The study has been registered in ClinTrials.gov under NCT01695408. A study report will be written upon completion of the study. Furthermore, results will be reported on ClinTrials.gov, and as peer-reviewed publication.

\section{Trial status}

The study was initiated by investigators (see authors); the legal sponsor is the GWT-TUD in Dresden. The study entered the field phase in November 2012, and has included 345 patients to date (10 December 2013). On an average, about 10-20 additional patients/month are recruited. Recruitment is planned until December 2014, and the field (documentation) phase of the study until December 2015. All study materials are available in English.

\section{Author affiliations}

${ }^{1}$ Department of Internal Medicine V, Comprehensive Pneumology Center, University of Munich and Asklepios Fachkliniken München-Gauting, Munich, Germany

${ }^{2}$ Department of Respiratory Medicine, Hannover Medical School, Hannover, Germany

${ }^{3}$ Department of Pneumology and Respiratory Critical Care Medicine, Thoraxklinik, University of Heidelberg, Heidelberg, Germany

${ }^{4}$ Department of Epidemiology, German Rheumatism Research Centre, Leibniz Institute, Berlin, Germany

${ }^{5}$ Department of Respiratory Medicine, University of Leipzig, Leipzig, Germany

${ }^{6}$ Medical Faculty, Institute for Clinical Pharmacology, Technical University, Dresden, Germany

Acknowledgements A summary of the study concept has been published in German language at the start of the registry. ${ }^{41}$ The authors are thankful for the project administration of Mrs Romy Hoppenz, GWT-TUD Dresden. 
Contributors All authors made substantial contributions to the design and coordination of the study. JB, DP, MMH, MK, JK and HW wrote the protocol. $\mathrm{DP}$ and JB wrote the present article, and all authors approved the final version to be published.

Funding The study is funded by an Unrestricted Educational Grant by Boehringer Ingelheim, Germany.

Competing interests None.

Patient consent Obtained.

Ethics approval The Ethics Committee of the Medical Faculty of the Technical University of Dresden, Germany approved the study protocol, the data collection forms and the patient information forms. All participating centres obtained approval of their local ethics committees.

Provenance and peer review Not commissioned; externally peer reviewed.

Data sharing statement No additional data are available.

Open Access This is an Open Access article distributed in accordance with the Creative Commons Attribution Non Commercial (CC BY-NC 3.0) license, which permits others to distribute, remix, adapt, build upon this work noncommercially, and license their derivative works on different terms, provided the original work is properly cited and the use is non-commercial. See: http:// creativecommons.org/licenses/by-nc/3.0/

\section{REFERENCES}

1. Raghu G, Collard HR, Egan JJ, et al. An official ATS/ERS/JRS/ ALAT statement: idiopathic pulmonary fibrosis: evidence-based guidelines for diagnosis and management. Am J Respir Crit Care Med 2011;183:788-824.

2. Coultas DB, Zumwalt RE, Black WC, et al. The epidemiology of interstitial lung diseases. Am J Respir Crit Care Med 1994:150:967-72

3. Gribbin J, Hubbard RB, Le Jeune I, et al. Incidence and mortality of idiopathic pulmonary fibrosis and sarcoidosis in the UK. Thorax 2006;61:980-5.

4. Raghu G, Weycker D, Edelsberg J, et al. Incidence and prevalence of idiopathic pulmonary fibrosis. Am J Respir Crit Care Med 2006; 174:810-16.

5. Scott J, Johnston I, Britton J. What causes cryptogenic fibrosing alveolitis? A case-control study of environmental exposure to dust. BMJ 1990;301:1015-17.

6. Iwai K, Mori T, Yamada N, et al. Idiopathic pulmonary fibrosis. Epidemiologic approaches to occupational exposure. Am J Respir Crit Care Med 1994;150:670-5.

7. von Plessen C, Grinde O, Gulsvik A. Incidence and prevalence of cryptogenic fibrosing alveolitis in a Norwegian community. Respir Med 2003;97:428-35.

8. Karakatsani A, Papakosta D, Rapti A, et al. Epidemiology of interstitial lung diseases in Greece. Respir Med 2009;103:1122-9.

9. Raghu G. Idiopathic pulmonary fibrosis. A rational clinical approach. Chest 1987;92:148-54.

10. Selman M, Carrillo G, Estrada A, et al. Accelerated variant of idiopathic pulmonary fibrosis: clinical behavior and gene expression pattern. PLoS ONE 2007;2:e482.

11. Song JW, Hong SB, Lim CM, et al. Acute exacerbation of idiopathic pulmonary fibrosis: incidence, risk factors and outcome. Eur Respir J 2011;37:356-63.

12. National Heart Lung and Blood Institute (NHLBI). Commonly used three-drug regimen for idiopathic pulmonary fibrosis found harmful (NIH News, 21. Oct 2011). http://www.nih.gov/news/health/oct2011/ nhlbi-21.htm (accessed 27 Jul 2013).

13. European Medicines Agency (EMA). Esbriet. CHMP Public Assessment Report EMA/CHMP/115147/2011. London, 2010. http:// www.ema.europa.eu/docs/en GB/document library/EPAR Public_assessment_report/human/002154/WC500103073.pdf (accessed 10 Dec 2013).

14. Richeldi L, Costabel U, Selman M, et al. Efficacy of a tyrosine kinase inhibitor in idiopathic pulmonary fibrosis. $N$ Engl J Med 2011;365:1079-87.

15. Koller $\mathrm{M}$, Neugebauer EA, Augustin $\mathrm{M}$, et al. [Assessment of quality of life in health services research-conceptual, methodological and structural prerequisites]. Gesundheitswesen 2009;71:864-72.

16. U.S. Department of Health and Human Services FDA Center for Drug Evaluation and Research. Guidance for industry: patient-reported outcome measures: use in medical product development to support labeling claims: draft guidance. Health Qual Life Outcomes 2006;4:79.

17. Szende A, Leidy NK, Revicki D. Health-related quality of life and other patient-reported outcomes in the European centralized drug regulatory process: a review of guidance documents and performed authorizations of medicinal products 1995 to 2003. Value Health 2005;8:534-48.

18. Institut für Qualität und Wirtschaftlichkeit im Gesundheitswesen. Allgemeine Methoden 3.0 vom 27.5. 2008. http://www.iqwig.de/ download/IQWiG_Methoden_Version_3_0.pdf (acessed 10 Dec 2013)

19. National Institute for Clinical Excellence (NICE). Guide to the methods of technical appraisal. London: NICE, 2004. http://www.nice.org.uk/ niceMedia/pdf/TAP Methods.pdf (accessed 10 Dec 2013).

20. Anonymous. SF-36. US Population Norm Values. 1998. http://www. sf-36.org/research/sf98norms.pdf (accessed 10 Dec 2013).

21. Morfeld M, Bullinger M, Nantke J, et al. [The version 2.0 of the SF-36 Health Survey: results of a population-representative study] Soz Praventiv Med 2005;50:292-300.

22. Hagaman JT, Kinder BW, Eckman MH. Thiopurine S-methyltransferase [corrected] testing in idiopathic pulmonary fibrosis: a pharmacogenetic cost-effectiveness analysis. Lung 2010;188:125-32.

23. Fioret D, Mannino D, Roman J. In-hospital mortality and costs related to idiopathic pulmonary fibrosis between 1993 and 2008 (abstract 1038A). Chest 2011;140.

24. Wilson JW, du Bois RM, King TE Jr. Challenges in pulmonary fibrosis: 8-the need for an international registry for idiopathic pulmonary fibrosis. Thorax 2008;63:285-7.

25. Alpert JS. Are data from clinical registries of any value? Eur Heart $J$ 2000;21:1399-401.

26. European Society of Cardiology. Registries, Databases, Protocols and Surveys. http://www.escardio.org/knowledge/ehs/registries/ (accessed 10 Dec 2013)

27. Hoeper MM, Huscher D, Ghofrani HA, et al. Elderly patients diagnosed with idiopathic pulmonary arterial hypertension: Results from the COMPERA registry. Int J Cardiol 2013;16:871-80.

28. Guenther A, Eickelberg O, Preissner KT, et al. International registry for idiopathic pulmonary fibrosis. Thorax 2008;63:841; author reply 841

29. European Commission. EurIPFnet European IPF Network: natural course, pathomechanisms and novel treatment options in idiopathic pulmonary fibrosis. http://ec.europa.eu/research/health/ medical-research/rare-diseases/projects/euripfnet_en.html (accessed 10 Dec 2013).

30. Glatz U. [GOLDnet-focus on rare lung diseases]. Dtsch Med Wochenschr 2011;136:p10.

31. Greiner W, Weijnen T, Nieuwenhuizen $\mathrm{M}$, et al. A single European currency for EQ-5D health states. Results from a six-country study. Eur J Health Econ 2003;4:222-31.

32. Brook RH, McGlynn EA, Cleary PD. Measuring quality of care-part two of six. N Engl J Med 1996;335:966-70.

33. Eakin EG, Resnikoff PM, Prewitt LM, et al. Validation of a new dyspnea measure: the UCSD Shortness of Breath Questionnaire. University of California, San Diego. Chest 1998;113:619-24.

34. Ries AL. Minimally clinically important difference for the UCSD Shortness of Breath Questionnaire, Borg Scale, and Visual Analog Scale. COPD 2005;2:105-10.

35. Kupferberg DH, Kaplan RM, Slymen DJ, et al. Minimal clinically important difference for the UCSD Shortness of Breath Questionnaire. J Cardiopulm Rehabil 2005;25:370-7.

36. Swigris JJ, Brown KK, Behr J, et al. The SF-36 and SGRQ: validity and first look at minimum important differences in IPF. Respir Med 2010;104:296-304

37. International Federation of Pharmaceutical Manufacturers and Associations (IFPMA). MedDRA (Medical Dictionary for Regulatory Activities). Internet: http://meddramsso.com/mssoweb/index.htm (accessed 10 Dec 2013)

38. Gitt AK, Bueno $\mathrm{H}$, Danchin N, et al. The role of cardiac registries in evidence-based medicine. Eur Heart J 2010;31:525-9.

39. Epstein M. Guidelines for good pharmacoepidemiology practices (GPP). Pharmacoepidemiol Drug Saf 2005;14:589-95.

40. Delgado-Rodriguez M, Llorca J. Bias. J Epidemiol Community Health 2004;58:635-41.

41. Behr J, Hoeper MM, Kreuter M, et al. [Characteristics and management of idiopathic pulmonary fibrosis: INSIGHTS-IPF registry]. Dtsch Med Wochenschr 2012;137:2586-8.

42. Quanjer PH, Tammeling GJ, Cotes JE, et al. Lung volumes and forced ventilatory flows. Report working party standardization of lung function tests, European Community for Steel and Coal. Official statement of the European Respiratory Society. Eur Respir J Suppl 1993;16:5-40. 


\section{Correction}

Juergen B, Hoeper MM, Kreuter M, et al. Investigating significant health trends in idiopathic pulmonary fibrosis (INSIGHTS-IPF): rationale, aims and design of a nationwide prospective registry. BMJ Open Resp Res 2014;1:e000010. doi:10.1136/bmjresp-2013-000010

The heading has changed from 'Pulmonary fibrosis' to 'Interstitial lung disease'.

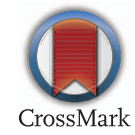

BMJ Open Resp Res 2014;1:e000010corr1. doi:10.1136/bmjresp-2013-000010corr1 